\title{
Incidence of different types of Myocardial Infarctions (MI) in diabetic and non-diabetic patients in cardiology unit of Lady Reading Hospital, Peshawar
}

\author{
Sumbal Jamil ${ }^{1}$, Mustajab Ghani ${ }^{2}$
}

\section{Submitted: \\ June 29, 2020 \\ Accepted: \\ August 6, 2020}

\section{Author Information}

${ }^{1}$ Institute of Paramedical Sciences

Khyber Medical University Peshawar

${ }^{2}$ Rehman College Allied Health Sciences

\section{Corresponding Author}

Sumbal Jamil

Institute of Paramedical Sciences

Khyber Medical University Peshawar

Email: sumbaljamil94@gmail.com

\begin{abstract}
Introduction: In past few decades prevalence of diabetes in patients with myocardial infarction has been remarkably increased. Diabetes mellitus has been well defined as a cardiovascular determinant in emergent nations. Myocardial infarction is the common cause of mortality and morbidity worldwide. Globally over 15.9 million myocardial infarction appeared in 2015. Cardiovascular events represent $80 \%$ of total deaths due to diabetes mellitus. Therefore, the objective was to determine the incidence of different types of myocardial infarctions in correlation with presence or absence of diabetes mellitus.

Material \& Methods: This was a cross sectional study carried out at Lady Reading Hospital Peshawar on 160 participants calculated by online calculator. Convenience sampling was applied, and subjects admitted in cardiology unit, CCU and emergency department of the hospital with the complaint of known myocardial infarction included. Pre-formed proforma was used to collect data from cardiology ward and CCU.

Results: Total of 160 subjects were included in this study. 101 subjects (63.1\%) were male while share of female subjects in the study was 59 (36.9\%). Mean age of the participants was $57.54 \pm 11.43$ (mean \pm S.D) and a range of 32-91 years. Diabetes was present in 85 (53.1\%) participants while other 75 participants (46.9\%) were non-diabetics. Anterior MI was seen in 36 diabetic subjects, inferior MI in 23, Lateral MI in 8, 14 were with extensive wall MI, 2 antero-lateral 2 \& infero-lateral MI in 2 diabetic subjects. Among non-diabetic participants, anterior MI was seen in 31 subjects, 11 had inferior MI, 14 were with lateral MI, 11 had extensive MI, 5 subjects showed antero-lateral and 3 were with infero-lateral MI of the heart. Conclusion: Incidence of MI was found more in diabetics as compared to non-diabetics. Anterior wall MI was commonest among diabetic participants.
\end{abstract}

The authors declared no conflict of interest and agreed to be accountable for all aspects of the work in ensuring that questions related to the accuracy or integrity of any part of the work are appropriately investigated and resolved.All authors contributed substantially to the planning of research, questionnaire design, data collection, data analysis and write-up of the article as part of a student research team at Institute of Paramedical Sciences Khyber Medical University Peshawar

This article may be cited as: Jamil, S., Ghani, M.Incidence of different types of Myocardial Infarctions (MI) in diabetic and non-diabetic patients in cardiology unit of Lady Reading Hospital, Peshawar. Rehman J Health Sci. 2020;2(1). 24-26

\section{INTRODUCTION}

Coronary Artery Disease (CAD) or Ischemic Heart Disease (IHD) is also an acquired heart disease among cardiovascular heart diseases. These are leading cause of death across the world. Ischemic heart disease is caused by atherosclerosis. Formation of plaque occurs in the inner walls of coronary arteries causing narrowing of the arteries and reducing the blood supply to the heart. ${ }^{1}$ As a result the heart muscles are devoid of oxygen and nutrients causing them to die. This results in myocardial infarction (MI) commonly known as heart attack. ${ }^{2}$ Cardiovascular diseases are the most usual and frequent result of mortality worldwide as in 2008 stated $30 \%$ of deaths. ${ }^{3}$ More than $75 \%$ of these are because of coronary artery diseases and stroke. Risk factors can be smoking, being overweight, high fats or cholesterol level and diabetes. ${ }^{4}$ The most common reason for coronary artery disease is the initiation of atherosclerosis. Plaque formation occurs within the coronary vessels. Stable plaque can only cause chest pain termed as angina and usually shortness of breath. A ruptured and unstable plaque causes the vessel to block completely leading to unstable angina and MI, the heart attack. ${ }^{5}$
The most well-known risk factors for ischemic heart disease are older age, active smoking, hypertension, diabetes and high cholesterol and lipoprotein levels. Coronary heart disease is associated with many risk factors as it is the major cause of myocardial infarction. Other determinants include male sex, less physical activity, family history and alcohol consumption. ${ }^{6}$ Management and prevention of acute coronary syndrome (ACS) is aimed against the determinants of reducing blood flow to the concerned area of heart muscle. This is accomplished with critical hospitalization and medical therapy consisting drugs that comfort chest pain and minimize the infarct size and also the drugs that hinders the formation of clot. Coronary angiography and percutaneous interventions are invasive measures applied to promote management of coronary arteries.

Diabetes mellitus is a type of metabolic diseases specified by high levels of glucose in the blood caused from abnormal insulin secretion, insulin action, and both. The persistent increased glycemic level is correlated with long-term damage, dysfunction and collapse of different vital organs, especially the kidneys, heart, eyes, nerves and blood vessels. ${ }^{8}$ 
Many procedures are involved in the pathogenesis of diabetes. These include the autoimmune destruction of the beta cells of the pancreas with resultant insulin shortening to pathologies that result in opposition to insulin activity. The lower action of insulin on target tissues leads the abnormalities in carbohydrates, proteins and fats. Improper insulin action leads to insufficient insulin production and decreased tissue feedback to insulin at complex pathways of hormone activity. ${ }^{8}$

Diabetic patients are at higher risk of developing MI which leads to cardiac failure and eventually death. ${ }^{9}$ A variety of cardiac alterations are associated with diabetes including left ventricular hypertrophy and reduced systolic and diastolic functions. ${ }^{10}$ There was scarcity of data available on the topic therefore, the aim of the study was to find out the relation among diabetes mellitus participants and occurrence of various kinds of myocardial infarctions.

\section{MATERIALS \& METHODS}

The study was conducted and completed at cardiology unit of Lady Reading hospital (Medical Teaching Institute) Peshawar. This was a cross-sectional study. The study was performed from September to December 2017 after proper approval from the head of the department of cardiology unit, Lady Reading hospital, Peshawar. The sampling technique which was adopted in order to carry out the study was convenient sampling. Subjects admitted in cardiology unit, CCU and emergency department of the hospital with the complaint of known myocardial infarction were taken as samples for the study. Participants were asked before about their will to take part in our study. The sample size can be calculated either using the formula or by using the sample size calculator. I used sample size calculator for calculation of my sample which came out to be a total of 160 . This sample size was used for completion of research study. A pre-formed proforma was designed for collection of data from the subjects. The Proforma had fifteen questions which were included after consulting with study supervisor and after studying existing materials and research papers published about this study title. The subjects included in this study were asked questions from proforma directly in cardiology wards, cardiac care unit and emergency department of Lady Reading hospital. The patients were asked direct questions and their response was noted in the given proforma. For some information required for the study were also obtained from examining subject's medical history and record, screening tests and diagnostic tests. Data was analyzed using descriptive statistics such as mean, standard deviation for continuous variables and number and percentage for categorical variables. Categorical variables were analyzed using Chi Square test. P value of Pearson's $<0.05$ was considered significant. All the statistical analyses were carried out by SPSS version 22 software.

\section{RESULTS}

The total participants of this observational cross-sectional study were 160 comprising of both male and female gender with different age. There was no limit of the age or gender in the study. The total male participants were 101 (63.1\%) while the female representation was 59 (36.9\%). The mean age was 57.54 with a standard deviation of \pm 11.434 ranging from $32-91$ years. Among total 85 diabetic participants in the study, 36 had MI of anterior wall, 23 were with inferior MI, 8 people had lateral wall MI, and, 14 participants exhibited extensive wall MI of the heart. 2 participants also showed MI of antero-lateral surface of heart while 2 others had MI of infero-lateral walls of the heart. Among the non-diabetic participants, 31 were with anterior wall MI, 11 had inferior MI, 14 were having lateral wall MI, 11 participants had extensive wall MI, and, 5 subjects with anterolateral while 3 others with infero-lateral MI also observed in the study. The Pearson's chi square test value for this relation of diabetes mellitus and type of MI occurred was 0.186 .

\section{Figure 1: History of Diabetes Mellitus \& Type of MI}

\begin{tabular}{|l|c|c|}
\hline Types of MI & \multicolumn{2}{|c|}{ Diabetes } \\
\hline & Yes & No \\
\hline Anterior MI & 36 & 31 \\
\hline Inferior MI & 23 & 11 \\
\hline Lateral MI & 8 & 14 \\
\hline Extensive MI & 14 & 11 \\
\hline Antero lateral MI & 2 & 5 \\
\hline Infero-lateral MI & 2 & 3 \\
\hline
\end{tabular}

We also want to know the incidence of different types of MI in both diabetics and non-diabetics in two genders i.e. male \& female participants of the study. Among females with diabetes mellitus, 15 had inferior MI and10 had anterior MI while in non-diabetic female participants, 7 had anterior MI and 5 had extensive walls MI. Figure 1

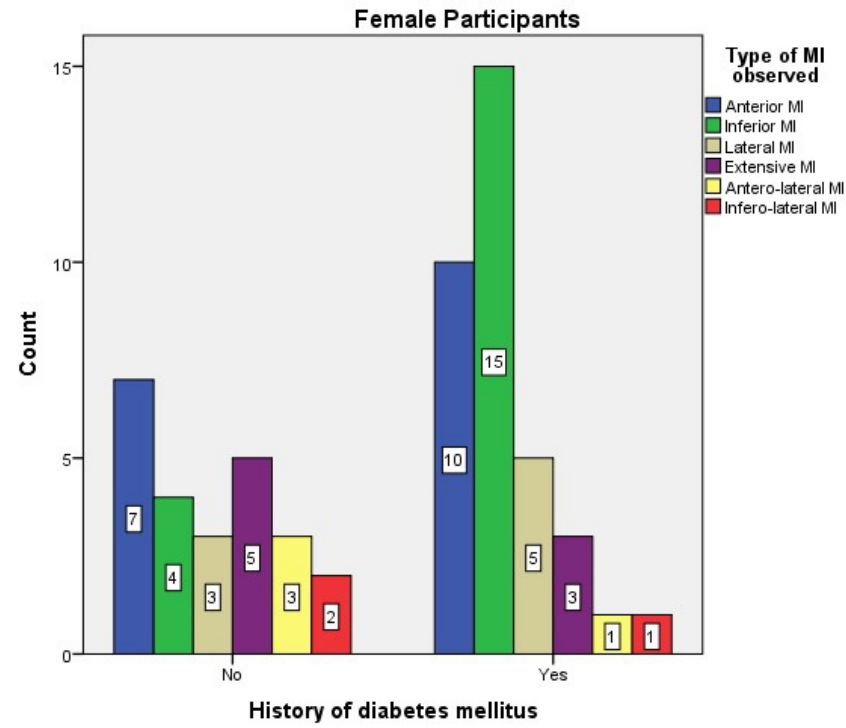

Figure 1: Type of MI seen in diabetic versus non-diabetic females 
Among male participants with diabetes, 26 had MI of anterior wall of the heart and 11 participants had extensive wall MI while 24 nondiabetics had anterior wall MI and 11 had lateral MI. Figure 2.

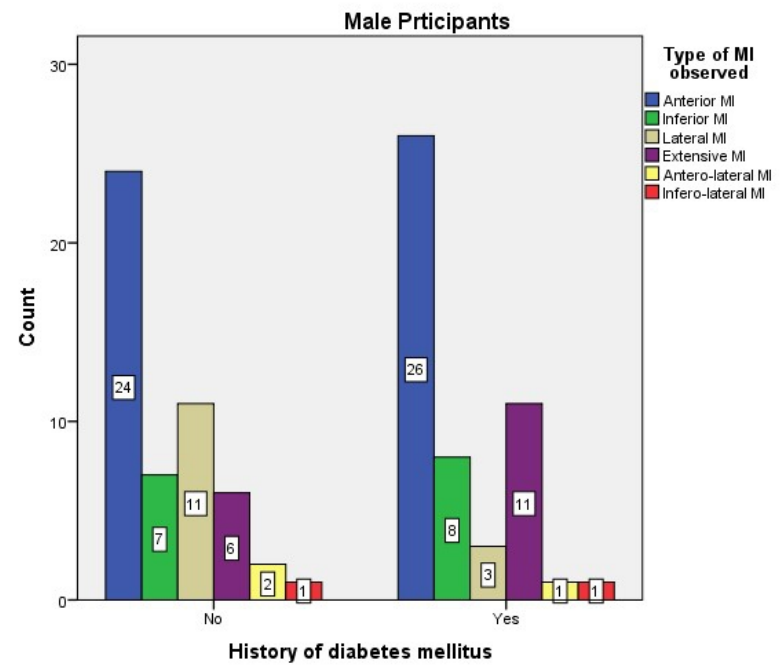

Figure 2: Type of MI seen in diabetic versus non-diabetic males

The association of type of MI occurred with diabetes in females was $p$-value $=0.91$ while with males was $p$-value $=0.259$.

\section{DISCUSSION}

The aim of the study was to find out the number of new cases of myocardial infarction (MI) in diabetic and non-diabetic patients. All the 160 subjects were recruited with presence of MI representing both male and female gender. The count of diabetic patients was found to be 85 (53.12\%) out of total 160. Among these diabetic patients 51 (60\%) were male and 34 (40\%) were female. The core objective of the study; finding incidence of different types of MI in diabetic and non-diabetic patients. This association turned to be insignificant after analysis of the data through different statistical tests. Out of total 67 participants with anterior MI occurrence; was $46.26 \%$ in non-diabetic while $53.7 \%$ of them were diabetic patients. M Agarwal et al., in his study had concluded high incidence of anterior wall involvement in acute MI in diabetic patients. For inferior wall MI occurrence, 32.35\% participants out of total 34 inferior MI patients were non-diabetic, and, remaining $63.64 \%$ were diabetic. Talking about total 22 lateral wall MI patients, $36.3 \%$ were diabetic and $63.6 \%$ were nondiabetic. Similarly out of total 25 extensive wall MI patients $56 \%$ were diabetic while other $44 \%$ patients were non-diabetics. ${ }^{11}$ We also reported occurrence of antero-lateral wall MI, out of total 7 only $28.5 \%$ were with diabetes and $71.4 \%$ were non-diabetic patients. Moreno et al., reported a large lipid levels with increased macrophage infiltration and occlusion in the atheroma of diabetic patients. Atheroma can then cause lead to occurrence of MI. ${ }^{12}$ This study established gender involvement in diabetes and type of MI occurred in them, but the association turned out non-significant in both males and females. In diabetic females; anterior MI occurred in $28.57 \%$, inferior MI was found in $42.85 \%, 14.28 \%$ presented lateral wall $\mathrm{MI}$ of heart, $8.57 \%$ occurrence of extensive wall MI, $2.85 \%$ females were seen with antero-lateral and another $2.85 \%$ with infero-lateral MI

\section{CONCLUSION}

This research study is concluded that the Incidence of anterior wall MI was highest in both diabetic and non-diabetic people. Anterior wall MI incidence was also highest in male subjects as compared to females. After anterior wall MI, second highest occurrence was seen in inferior wall MI of the heart. In diabetics participants it followed anterior MI in occurrence while in non-diabetic people MI of lateral wall of the heart was noticed higher in occurrence. Inferior wall MI was observed more common in female gender. Incidence of inferior wall MI was prominent in female diabetic people. The least common incidence was noticed of infero-lateral wall involvement in MI of the people.

\section{REFERENCES}

1. Alsalamah M. Heart Diseases Diagnosis Using Artificial Neural Networks: Coventry University; 2017.

2. Gaziano TA. Cardiovascular disease in the developing world and its cost-effective $\mathrm{m}$ a $\mathrm{n}$ a g e m e n t. C i r c u I a t i o n. 2005;112(23):3547-53.

3. Salehi-Abargouei A, Maghsoudi Z, Shirani F, Azadbakht L. Effects of Dietary Approaches to Stop Hypertension (DASH)style diet on fatal or nonfatal cardiovascular diseases-incidence: a systematic review and meta-analysis on observational prospective studies. Nutrition. 2013;29(4):611-8.

4. Okrainec K, Banerjee DK, Eisenberg MJ. Coronary artery disease in the developing world. American heart journal. 2004;148(1):7-15.

5. Doherty M, Ralston S. Davidson's Principles and Practice of Medicine. Systemic Connective Tissue Disease. 2010;25:1111.

6. Colledge NR, Walker BR, Ralston S, Davidson S. Davidson $s$ principles and practice of medicine: Churchill Livingstone/Elsevier; 2010.

7. Nyberg ST, Fransson El, Heikkilä K, Ahola K,
Alfredsson L, Bjorner JB, et al. Job strain as a risk factor for type 2 diabetes: a pooled analysis of 124,808 men and women. Diabetes care. 2014;37(8):2268-75.

8. Amsterdam EA, Wenger NK, Brindis RG, Casey Jr DE, Ganiats TG, Holmes Jr DR, et al. 2014 AHA/ACC guideline for the management of patients with non-STelevation acute coronary syndromes: executive summary: a report of the American College of Cardiology/American Heart Association Task Force on Practice Guidelines. Circulation. 2014;130(25):235494.

9. Booth G, Kapral M, Fung K, Tu J. Relation between age and cardiovascular disease in men and women with diabetes compared with non-diabetic 208 Per Løgstrup Poulsen and Johan V. Poulsen people: a population-based retrospective cohort study. Lancet. 2006;368:29-36.

10. Solomon SD, Sutton MSJ, Lamas GA, Plappert T, Rouleau JL, Skali H, et al. Ventricular remodeling does not accompany the development of heart failure in diabetic patients after myocardial infarction. Circulation. 2002;106(10):12515.
11. Agarwal M, Trivedi J, Sinh P, Dalal P, Saran R. Depression in patients of myocardial infarction-a cross-sectional study in northern India. J Assoc Physicians India. 2011;59:636-8.

12. Moreno PR, Murcia AM, Palacios IF, Leon MN, Bernardi VH, Fuster Vn, et al. Coronary composition and macrophage infiltration in atherectomy specimens from patients with diabetes mellitus. Circulation. 2000;102(18):2180-4. 